\title{
Developing Virtual Equipment to Enhance Learning of Structures and Material Science in an Aeronautics and Astronautics Engineering Program
}

\author{
Genisson Silva Coutinho ${ }^{1 *}$, Alberto S Mello², Alejandra J Magana ${ }^{3}$, Vinicius Dias ${ }^{4}$ and Vinicius Côrtes ${ }^{4}$ \\ ${ }^{1}$ Department of Mechanical and Materials Technology, Federal Institute of Bahia, Brazil \\ ${ }^{2}$ Embry-Ridle Aeronautical University, Florida \\ ${ }^{3}$ Computer and Information Technology, Purdue University, USA \\ ${ }^{4}$ Algetec Corporation, Brazil
}

*Corresponding author: Genisson Silva Coutinho, Department of Mechanical and Materials Technology, Federal Institute of Bahia, Brazil \& Engineering Education, Purdue University, West Lafayette, USA.

Received Date: February 16, 2020

Published Date: February 20, 2020

\section{Abstract}

This working paper presents the process of revamping a traditional hands-on laboratory course in an Aeronautics and Astronautics engineering program using a virtual equipment approach. Six traditional hands-on experiments will be used in a pilot program that aims to create virtual counterparts for each real experiment in the Aeromechanics course. The authors present one of these six experiments, and also discuss the main concerns and difficulties in developing the virtual equipment platform.

\section{Introduction}

Laboratory education represents an essential component of engineering curricula [1]. Laboratories help students to learn concepts, principles, and also fundamental engineering skills such as designing, problem-solving, and troubleshooting [2]. Despite this role, a series of constraints may prevent the ideal use of laboratories in engineering education. For example, traditional hands-on laboratories usually require significant investment in equipment, infrastructure, and space [3,4]. This fact may prevent universities and departments from creating the ideal lab environments necessary to students' learning of knowledge and skills [5]. To fix the issues caused by these constraints, educators are adopting emerging technologies that make the lab more inclusive, creative and effective. Among these emerging technologies, virtual laboratories are becoming pervasive in engineering education $[6,7]$.

Virtual labs have numerous affordances that make its use highly appreciated by educators and institutions. First, they usually require lower investment and fewer resources [8]. Second, they allow remote access, are accessible to students with disabilities and are safe to students [9]. Third, virtual labs enhance students conceptual understanding through the use of simulations and representations of abstract phenomena [10]. Finally, virtual labs usually are flexible and allow students to change the values of the different variables and explore the experimental results in a faster way than hands-on or remote experimentation [11]. A common critique regarding the use of virtual labs refers to use of idealized data that usually do not reflect the uncertainties and nuances of the real world [12]. Also, these labs usually lack the sense of reality necessary to immerse students in more authentic experiences [13]. Furthermore, a great deal of virtual labs focuses on the development of students' conceptual understanding about a particular phenomenon or theory [6]. To do this, the developers of such environments focus on reproducing or simulating the behavior of those phenomena, or systems, in the face of different variables. Students may vary the different variables and analyze how the system behaves. In most cases, characteristics associated 
with the equipment, the setup, the environment, as well as, all the experimental procedures are neglected. This approach is appropriated in most science-based courses and usually results in equivalent learning gains compared to traditional hands-on labs [6]. However, the development of important skills associated with the use of labs in engineering education [2] might be hindered by such approach. Among these skills, one might cite communication and collaboration, safety, designing of experiments, and learning from failure.

The development of virtual equipment uses the power of different technologies to create virtual apparatus that resemble real equipment, not only regarding visual representation but also regarding the operational procedures and physical behavior. Technologies such as a $3 \mathrm{~d}$ CAD, virtual reality (VR), finite element method (FEM), computational fluid dynamics (CFD), and game platforms, among others may be used simultaneously to create such virtual equipment. The use of virtual equipment preserves the main affordances of the common virtual labs and adds a higher level of visual fidelity between the virtual and real equipment, the possibility of practice in procedural knowledge associated with the use of engineering equipment, incorporation of real and simulated data, and the possibility of collaboration between students. In this paper, the authors present the process of revamping a traditional hands-on laboratory course in an Aeronautics and Astronautics engineering program using the virtual equipment. Six traditional hands-on experiments will be used in a pilot program that aims to create virtual counterparts for each real experiment in the course. The authors present one of these six experiments, and also discuss the main concerns and difficulties in developing the virtual equipment platform.

\section{A Learner-Centered Design Approach to Virtual Experimentation}

The design of the virtual equipment and corresponding learning materials have the student at its core; therefore, we followed a learner-centered approach [14]. For this, the design of the learning materials embeds scaffolding methods that account for prior knowledge of the learning domain, as well as motivational aspects. Scaffolding approaches are embedded in the design of the task (i.e., learning materials), the tools (i.e., the virtual equipment), and the interfaces. Specifically, scaffolding is provided in the form of guidance, so students clearly understand the goal of the assignment, and the design of the experiments by following principles of inquiry learning [15] along with articulation and reflection prompts that promote students' argumentation skills [16]. In addition, to foster student motivation, the whole content of the course, as well as the assignments, connect theory and practice in the context of Aeronautics and Astronautics Engineering.

\section{The Aeromechanics Course}

The students entering AAE as sophomores have to transition from a wider view of mathematics foundation and physics principles to the application of engineering analysis, technical communication, team-based work, and open-ended problem-solving. AAE204L is the first required laboratory course for AAE undergraduates and the only mandatory lab course in structures, a key area in aerospace engineering. Laboratory exercises provide a broad knowledge of the behavior of aerospace materials, as well as motivate students towards advanced studies in the subject. Recent evaluations show that students need to be motivated by these laboratory practices, by improving experiments' objectivity and context. To improve student learning and motivation, and successfully teach a rapidly increasing number of AAE undergrads, the Curriculum Committee has recommended the strategic use of web-based enhancements including virtual labs and online instruction.

\section{The Physical Experiments}

AAE204L is composed of six main laboratory experiments. In the first laboratory practice, students learn how sensors are used to obtain material properties, in a pre-design phase, and to determine the level of strain in an operational aerospace structure. The course material covers electrical resistance strain gages, Wheatstone bridge, and commercial strain indicators. Hands-on activities also include load transducers and extensometers. In the second practice, students learn how to obtain the basic mechanical properties of materials following test standards. The goal is to lead students through the process of obtaining stress-strain curves and determining Young's modulus, poison ratio, yield strength, ultimate strength, breaking strength, and ductility (elongation and reduction of area). In the laboratory experiment three, students learn about internal forces of aerospace structures. They deal with a simple truss structure configuration, where they have to measure strains in the structural members and use the material behavior learned from laboratory two to determine the axial load in each member. The measurements are compared with the values from truss theory, and simplifying assumptions are discussed. Laboratory four explores beam theory, in the context of aerospace structures. Detailed measurements are taken from several positions of a bending beam, where students have to check the applicability of classic theory by computing and measuring strains and deflections. In experiment 5 , students explore torsion loads in space structures. Rotors and drive shafts of helicopters, and airplane stabilizer torque tubes are typical cases where this type of loading mode is dominant. Also, fuselages and wings are subjected to torsion due to asymmetric loads. Students initially explore the concept of simple shear and then learn how to measure shear strain-based transformation of miss-oriented axial strains. Finally, in laboratory practice six, students learn about the multiaxial state of stress and check the theory for tensor transformation in several system orientations. Using a pressure vessel, students evaluate the thinwalled theory by calculating the principal stresses, determining the correspondent strains, and correlating them with the measured strains in the longitudinal and hoop directions.

The addition of virtual labs to the physical labs allows students to practice an experiment and understand concepts and 
safety procedures beforehand. It also increases the efficiency of student and instructor time in physical labs and decreases strain on laboratory equipment and scheduling. Furthermore, a Virtual Laboratory Learning Environment (VLLE) efficiently delivers selfstudy learning materials, allowing students to revisit theory and the fundamentals of each experiment. Complementarily, the use of the VLLE increase opportunities for scalability and for increasing the balance of virtual components. It is appropriate for distance education and fosters student's collaboration.

In the 2018 Spring semester, a series of virtual labs will be used as pre-laboratory lab assignments where students will perform similar experiments as the hands-on lab activities. Indeed, we may argue that these virtual labs work as scaffolding tools to the physical labs. As a way to foster student development of transfer skills, the experimental setups in the virtual labs will be slightly different than that of the hands-on labs but preserving the essence of each experiment. These virtual labs will focus on developing students' conceptual understanding of the theory and foster the development of procedural knowledge associated with the use of the experimental equipment. Furthermore, safety procedures will be evident in all the virtual setups. It is also worth mentioning that, data analysis will be required in all virtual labs. The following sections will present the development of the virtual laboratory learning environment created to support the use of virtual labs and describe the main steps toward the design of the virtual equipment used in the virtual experiment two: Determination of Mechanical Properties of Materials.

\section{The Virtual Laboratory Learning Environment}

Virtual Learning Environments (VLEs) are computer-mediated systems that enable online communication and collaboration between different actors in a learning process [17]. Furthermore, an effective VLE must provide a series of functionalities including noticeboard, online content delivery, assignment/quizzes, synchronous collaboration, and resources repository, among others [18]. Following these recommendations, a Virtual Laboratory Learning Environment was designed to support and enhance students' laboratory learning experiences.

The VLLE provides an online space where faculty, Teaching Assistants (TAs), and students share information and collaborate during the course. The environment was designed using a WordPress Learning Management System (WPLMS), and it was customized to make it easy for staff and students. The WPLMS is an open-source Learning Management System (LMS) which provides a standard set of functionalities and resources required by most virtual learning environments. To customize the VLLE, all the LMS functionalities that did not support the aims and scope of the aeromechanics course were removed. It made the interface clearer and easier to explore. Figure 1a shows the common interface for students and staff. Users must login and, depending on their privileges, different functionalities are available. Student privileges enable learners to explore the content and resources provided by instructors and Tas. They have to go through the sequence of content and quizzes to have access to the virtual lab environment (Figure 1b). TA privileges enable the teaching assistants to control and collaborate with the students, add, review and grade lab assignments and quizzes, and generate overall reports to the leading instructor. Finally, instructor privileges enable them to manage the system. A complete set of learning analytics functionalities $[19,20]$ will be available to instructors, providing additional information necessary to optimize the whole environment and the learning activities.

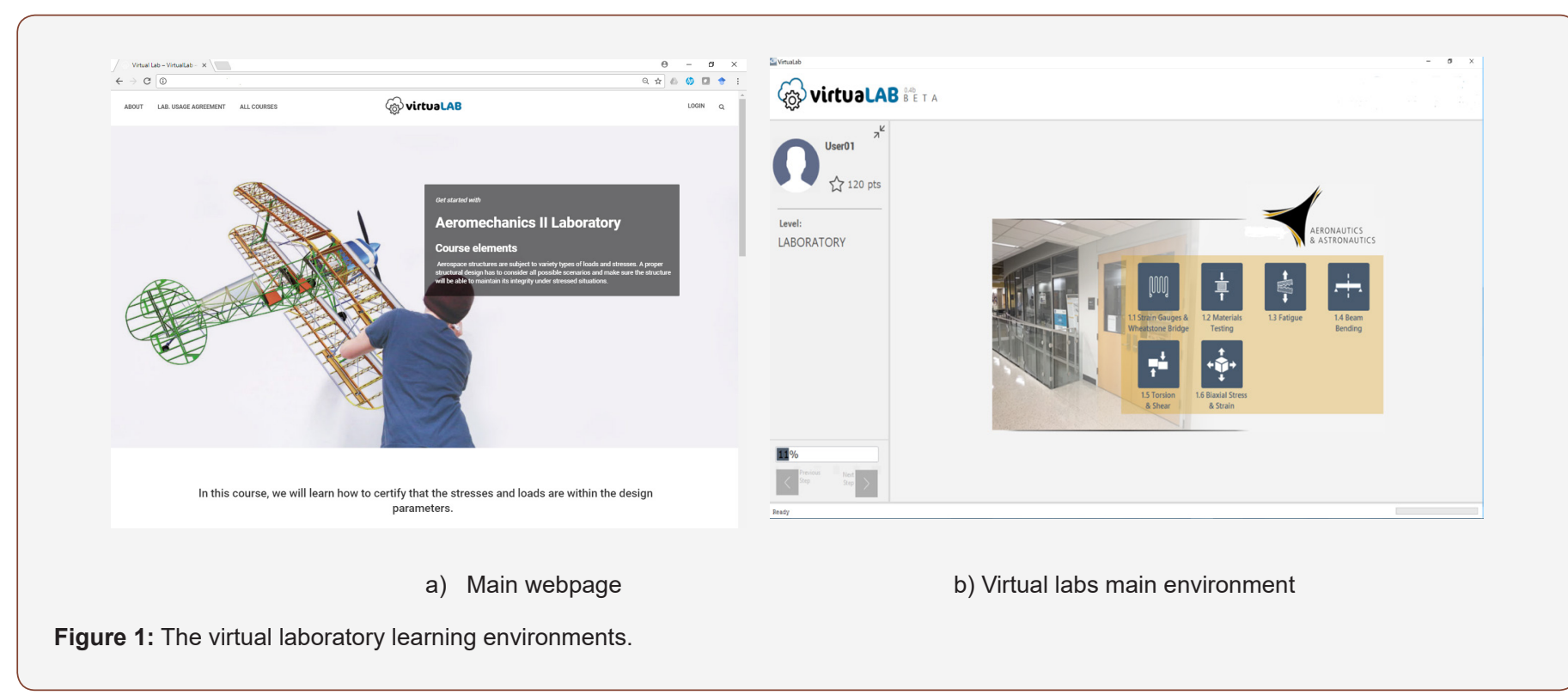

The AAE204L Virtual Laboratories: Development and Implementation

Potkonjak et al. [8] proposed the following criteria for evaluation of virtual laboratories in STEM education: "operating a virtual laboratory for a student must feel like they are working with real authentic devices in a real authentic space." (p. 311) Also, these authors unfolded the criteria mentioned above to consider the user interface, the virtual system behavior, the visualization, 
and communication and collaboration affordances. The user interface and the system behavior for each virtual equipment must correspond to the physical device or apparatus. The visualization must create a sense of authenticity to make students feel like operating a real device. Finally, the virtual environment must allow easy communication and collaboration among students, and between students and staff. In addition to these requirements, and following a learner-centered design approach, scaffolding tools were integrated into the system to provide different ways to introduce the content and activities, and also to guide students during initial stages of the experiments.

\section{Main Challenges}

The development of virtual equipment has numerous challenges. These challenges arise due the aims and scope of virtual equipment that go beyond just developing students' conceptual understanding. Using virtual equipment in the context of 3D virtual labs create a greater sense of reality and immersion and allow students to learn additional skills such as design and procedural skills. Thus, the development process must consider each component of the experimental apparatus, and model how they behave independently and collectively. This approach led to the following challenges: a) creating 3D cad counterparts for each real equipment; b) use textures and materials to improve the sense of reality; c) add properties and functionalities to each equipment including bottoms, keys, screens, movement, and others; d) associate such properties and functionalities to the behavior of real components; e) add simulations and physical characteristics; and f) integrate all components of the experimental apparatus and make them reproduce a real experiment. Each of these challenges may be addressed by one or more digital technologies, including
3D CAD software such as Solid works, Solid edge, and Blender, game engines such as Unity and Unreal, Multiphysics software such as Abaqus and Ansys, and integrated suites such as Labview. The next section describes how some of these digital technologies were used in the development of one of the six lab experiments of the Aeromechanics course. The proposed experiment aims to help students to learn how to determine mechanical properties of materials using tensile testing machines.

\section{Creating a Virtual Universal Testing Machine}

In AAE204L, activity two helps students to learn how to determine mechanical properties of materials. Students have to plan and conduct experiments using a UTM to perform tensile tests. The typical experimental procedure is well detailed in the laboratory manual and includes measurement of the specimen dimensions, identification of its material specification, clamping the extensometer to the body of the specimen, and checking if it is working properly. After that, the UTM is turned-on, and the load cell is calibrated. Then, the specimen is placed, aligned and clamped by the two machine grips. Finally, the specimen is loaded for its failure. Data from the load-cell and extensometers are used to create a load-elongation curve that, after some calculations, generates a stress-strain curve characteristic of the material under test. During the experiments, students have a limited time to perform different tests with the specimen and a great part of this time is spent on trying to learn how to operate the UTM, instead of focusing on the richness of the experiment. To overcome this limitation, and leveraging student's ability to perform tensile tests, a virtual UTM was created with similar characteristics of the actual machine (see Figures $2 \mathrm{a} \& 2 \mathrm{~b}$ ). All the experimental procedures were replicated using this virtual UTM.

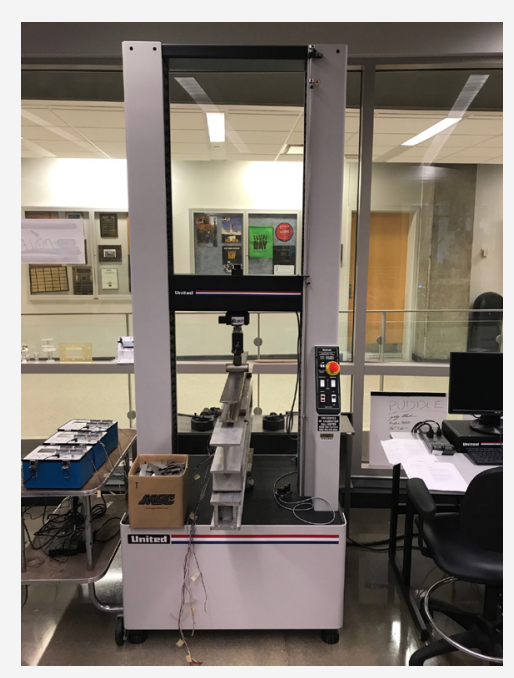

a) Actual UTM

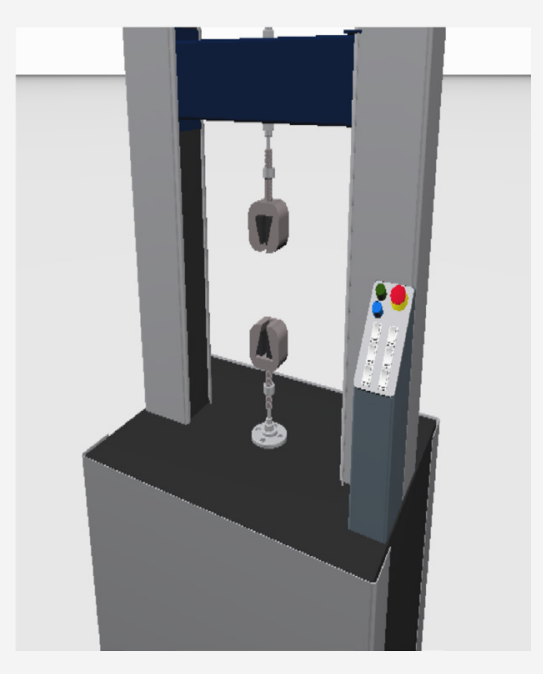

b) Virtual UTM

Figure 2: Universal Testing Machines.

Following similar steps described by Kang et al. [21], the development of the virtual UTM involved six major actions. First, a three-dimensional model of the actual machine was built using commercial 3D CAD software. This step was straightforward and had minor difficulties. Second, the experimental procedures were reviewed and complemented to switch the activity from a cookbook 
approach to a more engaging and challenging set of activities. Third, the 3D model was imported to a virtual reality package, and different controllers and functions were added to it, including commands to move the grips and wedges, translate the upper head, and measure load and displacement. Fourth, different tensile tests were simulated using a commercial finite element software. These simulations were linked with the experimental curves associated with the different materials. Fifth, an interface was created to integrate the simulations from the finite element software with the virtual UTM. Also, 3D manipulative functions were incorporated into that interface. Finally, scaffolding tools were developed to support students' learning.

\section{The Virtual UTM Interface}

The interface of the virtual UTM is illustrated in Figure 3a. Students may interact with the virtual machine in two different modes. The first mode also called "guided mode" provides a series of scaffolding tools that guide the students during the experimental procedures. When running in a guided mode, students learn stepby-step about of the specimen, machine operation, experimental setup and data analysis. Three basic virtual experiments were implemented to prepare students with the theoretical foundations and also the operational principles associated with the physical labs. These experiments are the classical rupture test, the determination of mechanical properties such as resilience and tenacity, and the determination of the modulus of elasticity and Poisson ratio.

In the classical rupture test (Figures $3 b$ \& 3c), students define the characteristics of the specimen, set the machine speed rate, clamp the specimen, and perform the test for its rupture. During the test, a finite element simulation is displayed, and the students are able to see how the specimen deforms. By loading and unloading the specimen, students can investigate how yielding and necking occur in different types of material. Guided experiment two aims to determine resilience and tenacity (see Figure 3d). Students follow the same steps as in the rupture test, but this time the system displays the curve load-elongation. Also, students can stop the loading cycle at any time to analyze the curve using a set of tools they allow students to calculate, for example, the area under the curve used to determine the resilience and tenacity. If during the test, students abort the experiment, the machine unloads the specimen, and a residual deformation may result as a consequence of yielding. Guided experiment three was designed to assist students with the physical lab that investigate ways to determine the modulus of elasticity and Poisson ratio. Basically, the UTM incrementally loads the specimen until a students' pre-defined load. At each load increment, the machine stops and asks students to take notes of the load, the elongation, and the transversal strain measured by a strain gage placed transversally in a specially constructed specimen. Students have to analyze the resulting data to determine the required properties.

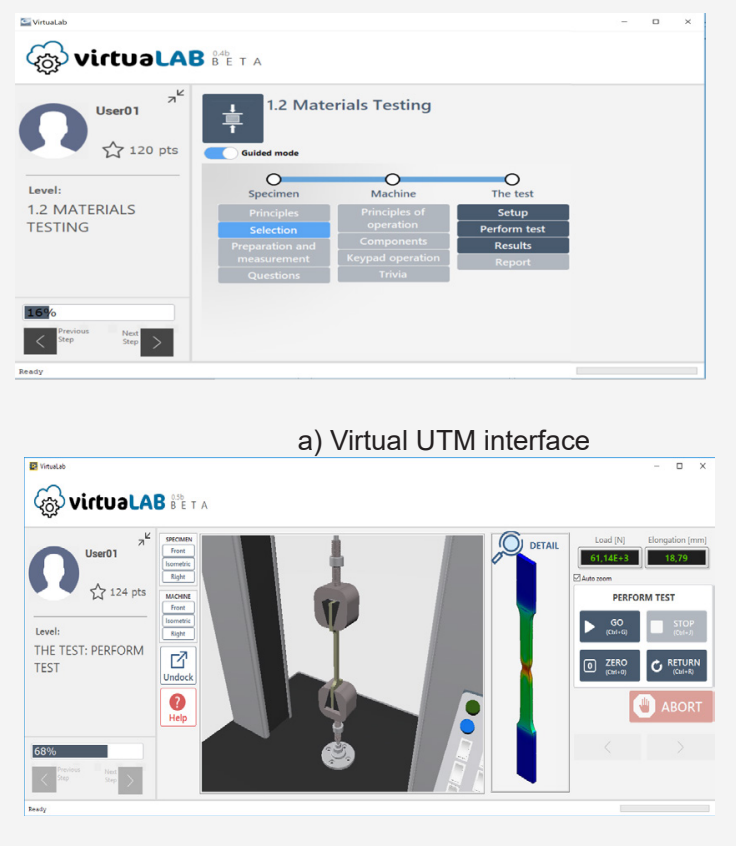

c) Rupture test displaying "necking"

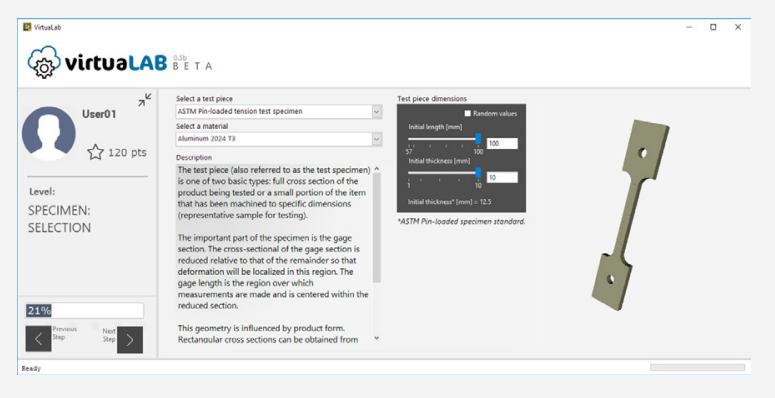

b) Rupture test - Guided mode

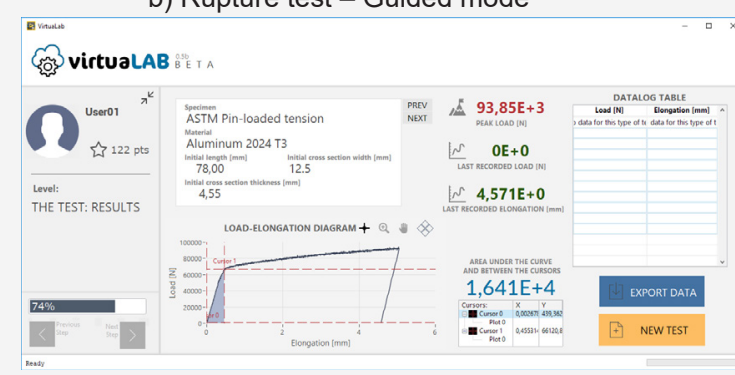

d) Guided experiment two interfaces

Figure 3: The virtual UTM interface and screens.

The second mode of operation, called "experimental" mode, simulates the real operation of the machine. No guidance is provided, and the results are stored at the end of the experiment for further analysis. In this mode, improper use of the machine may lead to wrong results, and students will have to identify and explain such results. 


\section{Future Developments}

Although most of the virtual laboratory experiments are still in a development process, and much has to be done in terms of identifying ideal digital technologies that support the development of authentic experiments, these authors have concerns regarding effective ways to assess the learning efficiency of the proposed virtual labs. For this reason, the new approach will be initially evaluated regarding accessibility, use, efficiency, accuracy, progression, and reflectiveness [22]. Accessibility will identify students' ability to activate and interpret all components of the equipment. Use will identify whether students engaged with the learning materials. Accuracy will identify how fast, easy and correctly students were able to achieve the learning task (i.e., experiments). Progression will assess how learners changed their response behaviors toward the learning experience as a whole (e.g., continuous engagement with it as they become more comfortable with the task), and reflectiveness will identify whether students reflected on their learning as they completed the experimentation procedures [23].

\section{Conclusion}

This is an ongoing project that aims to motivate and engage students in an aeromechanics laboratory course. The use of virtual environments and virtual equipment is an efficient way to foster students learning, but much care has to be taken when designing and implementing such virtual tools. Research shows that virtual labs must be authentic representations of the real experiments to be effective, and scaffolding tools must guide students during their initial steps. In this work, we presented one of six virtual laboratories that are currently under development in the Department of Aeronautics and Astronautics at a large midwestern university. Initial feedback from faculty and students are highly positive, however rigorous evaluation will be conducted to support continuous improvement.

\section{Acknowledgement}

None.

\section{Conflict of Interest}

No conflict of interest.

\section{References}

1. Coutinho GS, Stites NA, Magana AJ (2017) Understanding faculty decisions about the integration of laboratories into engineering education. In 2017 IEEE Frontiers in Education Conference (FIE). Indianapolis, IN, USA, pp. 1-9.

2. Feisel LD, Rosa AJ (2005) The Role of the Laboratory in Undergraduate Engineering Education. Journal of Engineering Education 94(1): 121130.

3. Bhargava P, Antonakakis J, Cunningham C, Zehnder AT (2006) Webbased virtual torsion laboratory. Computer Applications in Engineering Education 14(1): 1-8.

4. Melkonyan A, Gampe A, Pontual M, Huang G, Akopian D (2014) Facilitating remote laboratory deployments using a relay gateway server architecture. IEEE Transactions on Industrial Electronics 61(1): 477485 .
5. Magin D, Kanapathipillai S (2000) Engineering students' understanding of the role of experimentation. European Journal of Engineering Education 25(4): 351-358.

6. Brinson JR (2015) Learning outcome achievement in non-traditional (virtual and remote) versus traditional (hands-on) laboratories: A review of the empirical research. Computers and Education 87: 218-237.

7. Heradio R, De La Torre L, Galan D, Cabrerizo FJ, Herrera Viedma E, et al. (2016) Virtual and Remote Labs in Education: a Bibliometric Analysis. Computers \& Education 98: 14-38.

8. Potkonjak V, Gardner M, Callaghan V, Mattila P, Guetl C, et al. (2016) Virtual Laboratories for Education in Science, Technology, and Engineering: a Review. Computers \& Education 95: 309-327.

9. Gomes L, Bogosyan S (2009) Current Trends in Remote Laboratories. Industrial Electronics, IEEE Transactions on 56(12): 4744-4756.

10. Olympiou G, Zacharias Z, Jong $T$ de (2013) Making the invisible visible: Enhancing students' conceptual understanding by introducing representations of abstract objects in a simulation. Instructional Science 41(3): 575-596.

11. Zacharia ZC, Olympiou G, Papaevripidou M (2008) Effects of experimenting with physical and virtual manipulatives on students' conceptual understanding in heat and temperature. Journal of Research in Science Teaching 45(9): 1021-1035.

12. Balakrishnan B, Woods PC (2013) A comparative study on real lab and simulation lab in Communication Engineering from students' perspectives. European Journal of Engineering Education 38(2): 159171.

13. Balamuralithara B Woods PC (2009) Virtual laboratories in engineering education: the simulation lab and remote lab. Computer Applications in Engineering Education 17(1): 108-118.

14. Soloway E, Guzdial M, Hay KE (1994) Learner-centered design: the challenge for HCI in the $21^{\text {st }}$ century. Interactions $1(2)$ : $36-48$.

15. Hancock C, Kaput JJ, Goldsmith LT (1992) Authentic inquiry with data: Critical barriers to classroom implementation. Educational Psychologist 27(3): 337-364.

16. Chen YC, Park S, Hand B (2016) Examining the use of talk and writing for students' development of scientific conceptual knowledge through constructing and critiquing arguments. Cognition and Instruction 34(2): 100-147.

17. Dillenbourg P, Schneider D, Synteta P (2007) Virtual Learning Environments. In Proceedings of the 3rd Hellenic Conference. Information \& Communication Technologies in Education, Kastaniotis Editions, Greece, pp. 3-18.

18. Britain S, Liber O (1999) A Framework for pedagogical evaluation of virtual learning environments. JISC Technology Applications, Vol (41).

19. Romero S, Guenaga M, Garcia Zubia J, Orduña P (2014) New challenges in the Bologna Process using Remote Laboratories and Learning Analytics to support teachers in continuous assessment. 2014 International Symposium on Computers in Education (SIIE), pp. 227-230.

20. Siemens G (2013) Learning Analytics: The Emergence of a Discipline. American Behavioral Scientist 57(10): 1380-1400.

21. Kang SC, Chan YC, Lu CY, Lai JS, Lee TH (2013) Development of virtual equipment: Case study of the Venturi tube experiment. Journal of Professional Issues in Engineering Education and Practice 139(4): 281289.

22. Quintana C, Krajcik J, Soloway E (2001) Exploring a description and methodology for learner-centered design. In WF Heineke \& L Blasi (Eds.), Methods of evaluating educational technology. Information Age Publishing.

23. Magana AJ, Silva Coutinho G (2017) Modeling and simulation practices for a computational thinking-enabled engineering workforce. Computer Applications in Engineering Education 25(1): 62-78. 